\title{
A Case of Pregnancy with Choriocarcinoma Complicated by a Cerebral Hemorrhage and Lung Metastasis
}

\author{
Yan $\mathrm{Wu}^{\mathrm{a}} \quad$ Peng Ren ${ }^{\mathrm{b}} \quad$ Jian Chen ${ }^{\mathrm{c}}$ Ling $\mathrm{Ai}^{\mathrm{d}}$ \\ aDepartment of Obstetrics and Gynecology, Women and Children Hospital of Jiaxing \\ University, Jiaxing City, China; bepartment of Pathology, Women and Children Hospital \\ of Jiaxing University, Jiaxing City, China; 'Department of Radiology, Women and Children \\ Hospital of Jiaxing University, Jiaxing City, China; ${ }^{d}$ Department of Obstetrics and \\ Gynecology, Women and Children Hospital of Jiaxing University, Jiaxing City, China
}

\section{Keywords}

Pregnancy with choriocarcinoma · Cerebral hemorrhage $\cdot$ Lung metastasis · Cancer and pregnancy

\begin{abstract}
Pregnancy with choriocarcinoma is a rare tumor. It is rare for neonates to survive the third trimester. This article reports the clinical data of a live fetal pregnancy with choriocarcinoma complicated by brain and lung metastases. The patient was admitted to the hospital for "menopause 28 weeks +5 days, dizziness with nausea and vomiting 2 days." After 5 hours of admission, the patient had sudden convulsions, urinary incontinence, and coma. A head computed tomography (CT) examination in the emergency department revealed a cerebral hemorrhage in the right occipital lobe and broke into the ventricular system with brain herniation. She was immediately transferred to the intensive care unit for the emergency cesarean section and intracerebral hematoma removal. A postoperative CT scan revealed that the tumor in the upper lobe of the right lung was considered lung cancer, with multiple metastases in both lungs. Postoperative pathology was metastatic choriocarcinoma tissue seen in the blood clot. Based on the pathological diagnosis of choriocarcinoma, a chemotherapy regimen was developed with 2 courses of EP regimen and 8 courses of combined EMA-CO chemotherapy regimen. The patients were followed up for 2 years and ultimately resolved.
\end{abstract}




\section{Case Reports in Oncology}

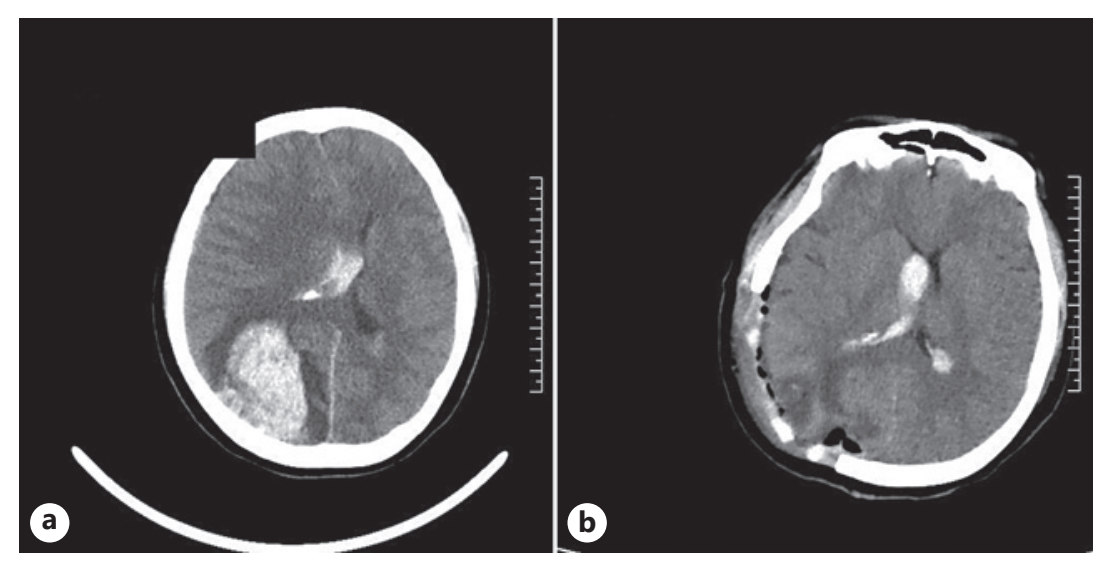

Fig. 1. Choriocarcinoma with brain metastases (a); Right occipital lobe hematoma removed after surgery (b).

\section{Introduction}

The incidence of choriocarcinoma is $0.02-0.05$ per 1,000 pregnancies in Europe and America and can reach $0.2-4$ per 1,000 pregnancies in Southeast Asia [1]. Before the advent of chemotherapy drugs, the mortality rate was over 90\% [2].The primary treatment for choriocarcinoma is chemotherapy, and the appropriate plan is determined by staging and grading. Accurate diagnosis of choriocarcinoma in pregnancy can significantly improve its prognosis at an early stage. This article reports a case of a 25-year-old woman in her third pregnancy and her first childbirth. She developed dizziness with nausea and vomiting at 28 weeks +5 days of gestation in the third trimester. After admission, she was diagnosed as pregnant with choriocarcinoma with brain metastasis and lung metastasis.

\section{Case Report}

The patient, female, 25 years old, Han nationality, from Jiaxing City, 0-0-2-0, was admitted to the hospital for "menopause for 28 weeks +5 days, dizziness with nausea and vomiting 2 days." The patient woke up from a nap the day before admission and developed dizziness, no optical rotation, no tinnitus, vomiting of stomach contents $>10$ times, accompanied by upper abdominal discomfort, no diarrhea and melena. The initial diagnosis in the emergency department was "dizziness to be investigated, $>6$ months of pregnancy."

Five hours after admission, the patient had sudden convulsions, urinary incontinence, and loss of consciousness. Physical examination showed coma, blood oxygen saturation $97 \%$, blood pressure 130/80 $\mathrm{mm} \mathrm{Hg}$, breath 18 times/min, fetal heart rate 130 $\mathrm{bpm}$, right pupil $6 \mathrm{~mm}$, the disappearance of light reflection, left pupil $5 \mathrm{~mm}$, weak light reflection, and unable to cooperate with muscle strength. The muscle tone of the extremities increased, and the Glasgow score was 5 points. According to symptoms and physical signs, physicians consider cerebral hemorrhage, brain herniation, and secondary epilepsy and prescribe sedation and spasmolysis to reduce intracranial pressure. Emergency head computed tomography (CT) showed (Fig. 1a): (1) right occipital lobe cerebral hemorrhage and break into the ventricular system, accompanied by brain herniation, need to consider the possibility of hemorrhagic cerebral infarction caused by venous sinus thrombosis; (2) a small amount of subarachnoid hemorrhageFig. 1. Admission diagnoses were as follows: (1) cerebral hemorrhage (right occipital lobe and cerebral artery malformation),

\section{Karger'}



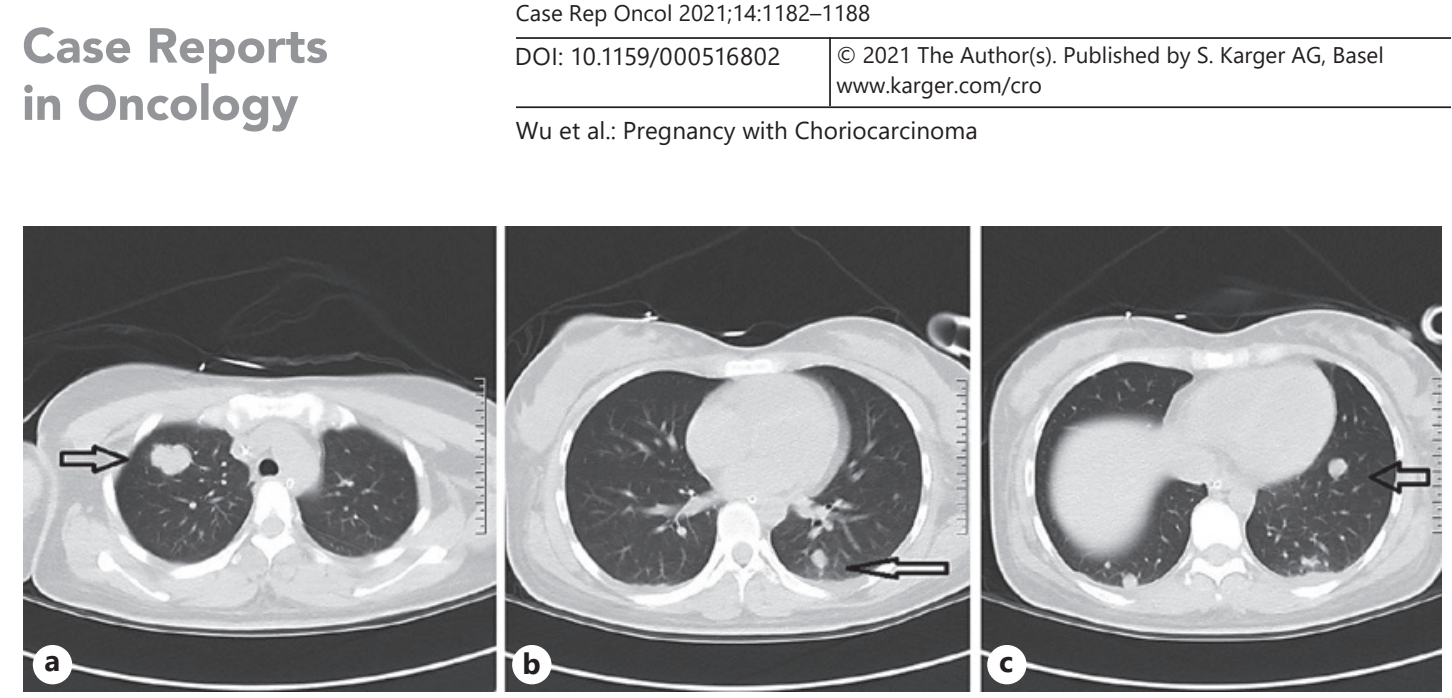

Fig. 2. a-c Postoperative review of chest CT, lump in the upper lobe of the right lung considered as lung cancer, multiple metastases in both lungs. CT, computed tomography.
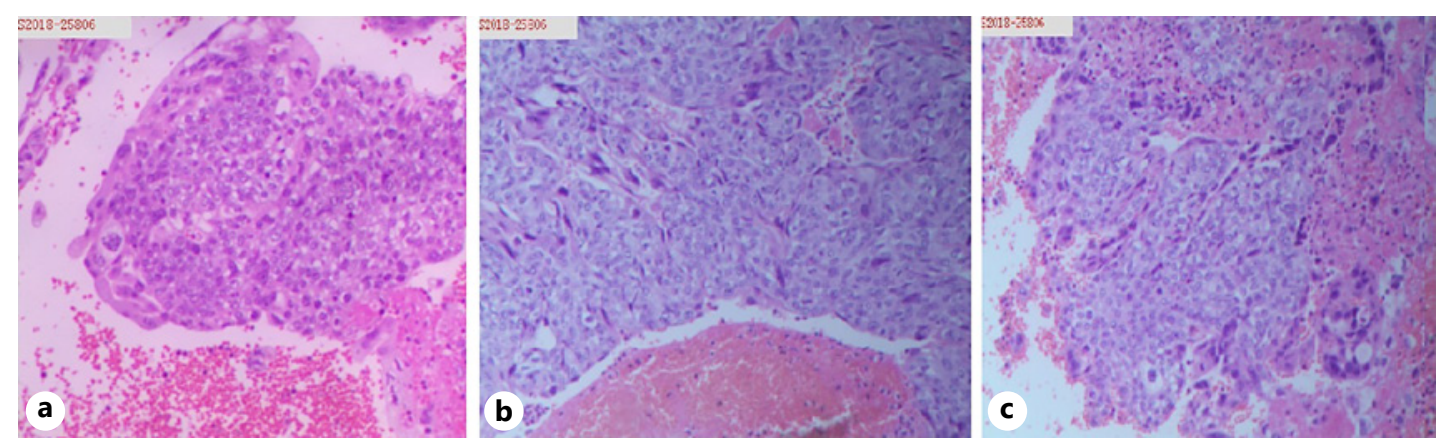

Fig. 3. a-c (Tissue in the hematoma cavity) Metastatic choriocarcinoma tissue was seen in the blood clot.

(2) cerebral hernia, (3) secondary epilepsy, and (4) pregnancy status (28 weeks +5 days of pregnancy).

\section{Treatment}

After multidisciplinary discussion, an emergency cesarean section was performed under general anesthesia. During the operation, a live boy was delivered in the LOT position (transferred to NICU after birth), Apgar 2-3-3, weight 1,180 G, and the placenta was delivered intact. During the operation, intracerebral hematoma removal surgery and decompressive craniectomy were performed simultaneously. The periosteal expansion was reduced to repair the dura, and the extradural indwelling rubber tube was used for drainage. Intraoperative diagnoses were as follows: (1) cerebral hemorrhage, (2) cerebral herniation, (3) G3 P1 28 weeks of pregnancy +5 days LOT dystocia live infant, (4) severe asphyxia of the newborn, and (5) premature delivery.

Postoperative blood transfusion corrects blood loss and coagulopathy. The CT examination report on the first day after surgery showed the following: (1) after cerebral hemorrhage, the right occipital lobe hematoma was cleared, and the cerebral ventricle was hemorrhage (Fig. 1b); (2) lump in the upper lobe of the right lung considered lung cancer, multiple metastases in both lungs (Fig. 2a-c). Postoperative pathology: (1) (tissue in the hematoma cavity) metastatic choriocarcinoma tissue was seen in the blood clot (Fig. 3a-c); (2) (cranial) bone tissue. Immunohistochemistry; 4 tablets: CK (pan)(+), HCG(+), and HPL(+) (Fig. 4a-c). The oncologist indi- 


\section{Case Reports in Oncology}

\begin{tabular}{l|l}
\hline Case Rep Oncol 2021;14:1182-1188 \\
\hline DOI: 10.1159/000516802 & $\begin{array}{l}\text { ○ 2021 The Author(s). Published by S. Karger AG, Basel } \\
\text { www.karger.com/cro }\end{array}$ \\
\hline
\end{tabular}

Wu et al.: Pregnancy with Choriocarcinoma
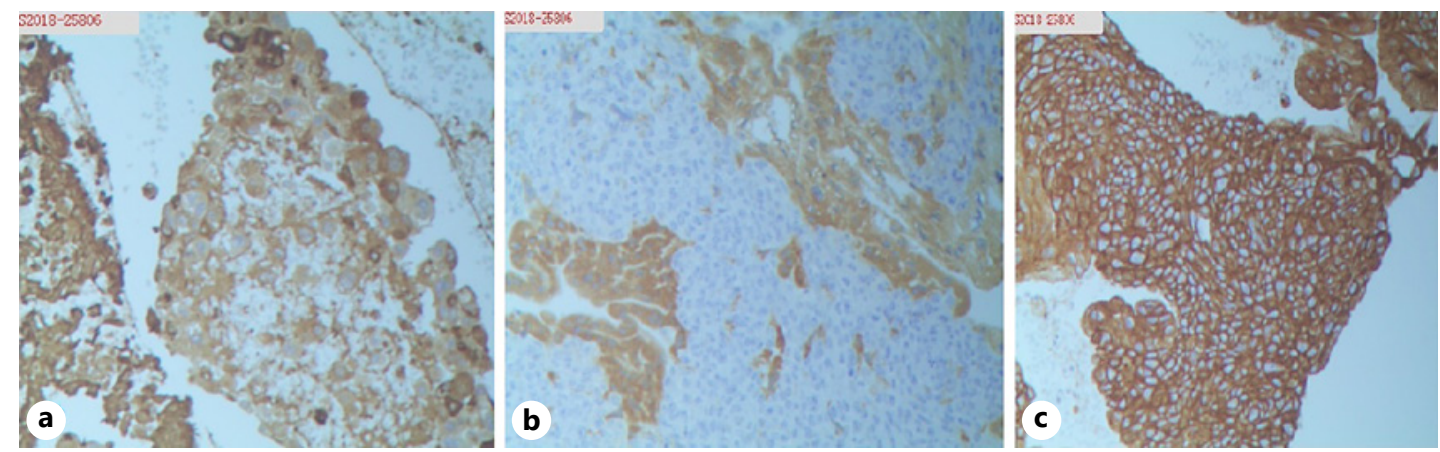

Fig. 4. a-c Immunohistochemistry of skull bone tissue: 4 slices: CK (pan)(+), HCG(+), and HPL(+).
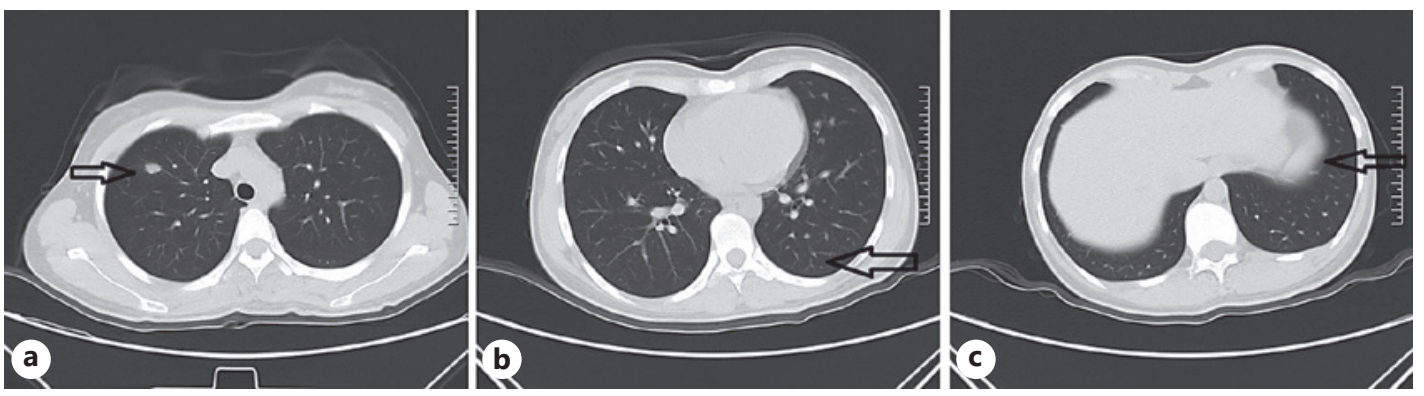

Fig. 5. a-c The metastatic lung lesions gradually subsided.

cated that the disease had entered stage 4. The risk score of choriocarcinoma was 16 points, indicating a very high risk, with lesions in the brain and lungs. First, choose the EP regimen for 2 courses and actively deal with the situation of brain surgery. The chemotherapy regimen for the first 1-2 courses is as follows: (1) etoposide injection $0.16 \mathrm{~g} \mathrm{qdx} 2 \mathrm{~d}$; (2) cisplatin injection $30 \mathrm{mg} \mathrm{qdx} 2 \mathrm{~d}$. After the first course of treatment, the patient's HCG dropped to a normal level $(<1.2 \mathrm{U} / \mathrm{L})$. The third course of treatment was combined with EMA-CO chemotherapy, and she was discharged after the third course. Discharge diagnoses were as follows: (1) choriocarcinoma (lung metastasis and brain metastasis), (2) pregnancy with cerebral hemorrhage (intracerebral hematoma removal + decompressive craniectomy), (3) pregnancy with brain hernia, (4) G3 P1 28 weeks gestation + 5 days LOT dystocia live baby, (5) neonatal asphyxia (severe), (6) premature delivery, and (7) the third postoperative chemotherapy.

\section{Outcome and Follow-Up}

The patient underwent a total of 2 courses of EP regimen + 8 courses of EMA-CO regimen. The metastatic lung lesions gradually subsided (Fig. $5-\mathrm{c}$ ). The HCG has been regular after the reexamination. The patient's condition was followed up for 2 years after discharge. HCG was regular, no distant metastasis or recurrence, and choriocarcinoma was in complete remission.

The condition of the newborn was as follows: the baby was admitted to NICU immediately after birth. When the baby was discharged, the gestational age was corrected for $36 \mathrm{~W}$ +4 days, the length was $46 \mathrm{~cm}$, the head circumference was $32 \mathrm{~cm}$, and the weight was 2,330 g. After 2 years of follow-up, HCG was regular. The Department of Neonatal and Child Health is included in the follow-up management of high-risk infants, and the child's body weight and height are all up to standard in the follow-up 2 years after birth. 


\section{Discussion/Conclusion}

The gestational trophoblastic tumor is a unique type of malignant tumor. Even with extensive metastasis, $90 \%$ of patients can be cured by chemotherapy alone. Sixty percentage of gestational trophoblastic tumor is secondary to a molar pregnancy, $30 \%$ is secondary to abortion, $10 \%$ is secondary to term pregnancy or ectopic pregnancy, and choriocarcinoma can be secondary to molar or secondary to non-mole pregnancy $[2,3]$. Pregnancy with choriocarcinoma is very rare clinically and pathologically. This case is a pregnancy with choriocarcinoma. There are a placenta and fetus in the uterus, combined with choriocarcinoma outside the genital tract. There was no lesion on the patient's uterus. The patient was young and required to preserve the uterus. The placenta was not routinely examined by pathology, and it was not clear whether the placenta had choriocarcinoma pathology. The patient developed extra-genital choriocarcinoma while the primary lesion disappeared. It is speculated that the reason may be that the primary lesion is degenerated and absorbed, the trophoblast cells are in a static state in the extrauterine blood vessels, and they become malignant due to endocrine stimulation, or part of the villi cells develop defects and become malignant, and then the blood transfers to the lungs and brain.

Vaginal bleeding is the most common symptom of choriocarcinoma. Bleeding from extrauterine organs such as the lung (60-75\%), liver (15-20\%), central nervous system (15-20\%), and gastrointestinal tract (10-20\%) may be those cases accompanied by tumor spread outside the uterus as the first symptom $[2,4,5]$. The prognosis of choriocarcinoma with brain metastasis is dangerous and is the leading cause of death. They are usually accompanied by lung metastasis or vaginal metastasis [6].The initial stage of metastasis is mostly asymptomatic. Brain metastasis is divided into 3 stages. The first stage is the tumor thrombosis stage, which manifests as transient cerebral ischemia symptoms, such as sudden falls, temporary aphasia, and blindness. The second stage is the brain tumor stage, with headache, dizziness, jet-like vomiting, hemiplegia, convulsions, and even coma. The third stage is the cerebral herniation stage. Due to the brain tumor's compression and the edema and bleeding of the surrounding tissues, the intracranial pressure further rises. The brain herniation forms oppress the center of life and cause death. This case first manifested as the third stage of herniation of brain metastasis, which is very dangerous. Immediate emergency craniotomy was performed to remove blood clots in the brain, relieve intracranial hypertension, and save the mother and the fetal life. Postoperative CT examination and pathological examination of the specimens were performed to obtain a pathological report to confirm the diagnosis and guide the postoperative treatment plan.

Choriocarcinoma is highly malignant and metastasizes early. Before the advent of chemotherapy drugs, the mortality rate was over $90 \%[2,3]$.The primary treatment method of choriocarcinoma is chemotherapy $[5,6]$. The appropriate plan is determined by staging and grading, and the comprehensive treatment of surgery and radiotherapy is appropriately coordinated. Early or low-risk patients can be treated with single-agent chemotherapy. Advanced, high-risk, and drug-resistant patients are mainly treated with combination chemotherapy, supplemented by local treatment $[7,8]$. If chemotherapy for liver or brain metastases or lesions with a diameter of $>5 \mathrm{~cm}$ is not sufficient, surgery or radiotherapy should be performed as soon as possible [1]. A single lesion can be operated on or radiotherapy and multiple lesions can be treated with chemotherapy. The chemotherapy regimen should be reasonable, adequate, and timely, and the principle of individualization should be emphasized in the treatment process. For low-risk patients (stage I, II, and III, risk score $\leq 6$ ), MTX or actinomycin D monotherapy is required. After HCG levels return to normal, consolidation chemotherapy must continue for 2-3 cycles to reduce recurrence risk $[3,9]$. The complete remission rate for low-risk patients is close to $100 \%$. High-risk patients (II, III, and IV, risk score $>6$ ) require a comprehensive multi-drug chemo- 
therapy regimen for treatment. The most common regimen is the EMA-CO regimen: Etoposide, MTX/Leucovorin, and Actinomycin D, followed by Cyclophosphamide and Vincristine [2, 10]. This program's complete remission rate is $67-78 \%$, and the 5 -year overall survival rate is $85 \%$ to $94 \%$. The prognosis of patients with liver and brain metastasis is low, and high-risk patients should receive 4 consolidation chemotherapy cycles. Resistant patients can receive wholebrain radiotherapy (total dose of 3,000 cGy) or follow stereotactic radiotherapy and chemotherapy [9-11].However, whether radiotherapy is more effective than chemotherapy is still controversial. In this report, the patient received 2 EP regimen courses + 8 courses of EMA-CO combined chemotherapy regimen, with a good prognosis and no recurrence 24-months followup. For patients with high intracranial pressure, especially those with brain herniation, surgery should be taken.

Accurate diagnosis of choriocarcinoma in pregnancy can significantly improve its prognosis at an early stage [11]. Although the incidence of choriocarcinoma in pregnancy is shallow, regular monitoring is necessary. All patients with choriocarcinoma should be closely followed up for 2 years after treatment, with strict contraception during the follow-up period. The patient, in this case, was treated as a high-risk pregnancy. The patient with stage IV had blood HCG once a month for 24 months. The most recent HCG was rechecked in December 2020 , and the condition was completely relieved.

\section{Statement of Ethics}

Written informed consent was obtained from the patient for the publication of this case report and any accompanying images. The Ethics Committee of the Women and Children's Hospital of of Jiaxing University has approved the research protocol. None of the content is included in another manuscript, has been published previously, or is currently under consideration for publication elsewhere.

\section{Conflict of Interest Statement}

The authors declare no potential conflicts of interest with respect to the research, authorship, or publication of this article.

\section{Funding Sources}

No.

\section{Author Contributions}

All authors participated substantively to the paper and have approved the final version of the manuscript. Yan $\mathrm{Wu}$ and Peng Ren involved in conception, preparation, and review of the manuscript; Ling Ai and Jian Chen involved in preparation and review of the manuscript.

\section{Data Availability Statement}

Data are available on request to the authors.

\section{Karger'}




\section{References}

1 Ganapathi KA, Paczos T, George MD, Goodloe S, Balos LL, Chen F. Incidental finding of placental choriocarcinoma after an uncomplicated term pregnancy: a case report with review of the literature. Int J Gynecol Pathol. 2010;29(5):476-8.

2 Wang X. Progress in diagnosis and treatment of trophoblastic diseases in pregnancy_王晓雨.pdf $>$. Chinese J Fam Plann Gynecol. 2019;11:6-7.

3 Jing Y. Progress in clinical research on gestational trophoblastic diseases. Chinese J Gynecol Obstetr. 2017; 33(2):225-6.

4 Strohl AE, Lurain JR. Postmolar choriocarcinoma: an independent risk factor for chemotherapy resistance in low-risk gestational trophoblastic neoplasia. Gynecol Oncol. 2016;141(2):276-80.

5 Song L, Li Q, Yin R, Wang D. Choriocarcinoma with brain metastasis after term pregnancy: a case report. Medicine. 2018;97(42):e12904.

6 Ren Z, Yu L, Xie M, Liang Y, Zhu F, Huang R, et al. Successful treatment of multisite hemorrhage by several methods in brain metastasis of choriocarcinoma: a case report. Medicine. 2018;97(21):e10794.

7 Ma D, Zhang Z, Li S. [Progress of the diagnosis and treatment of pulmonary metastasis of gestational choriocarcinoma]. Zhongguo Fei Ai Za Zhi. 2011;14(10):801-5.

8 Lee E, Cho H. A case of intraplacental choriocarcinoma with pulmonary metastasis. Case Rep Oncol. 2019; 12(3):802-6.

9 Jung S-H, Choi YJ, Kim MS, Park H-C, Han M-R, Hur SY, et al. Distinct genomic profiles of gestational choriocarcinoma, a unique cancer of pregnant tissues. Exp Mol Med. 2020;52(12):2046-54.

10 Jiao L, Ghorani E, Sebire NJ, Seckl MJ. Intraplacental choriocarcinoma: systematic review and management guidance. Gynecol Oncol. 2016;141(3):624-31.

11 Horowitz NS, Berkowitz RS, Elias KM. Important insights for non-molar choriocarcinoma. BJOG. 2020;127(9): 1108. 